\title{
Extensive practice does not eliminate human switch costs
}

\author{
GiJsbert Stoet \\ University of Leeds, Leeds, England \\ AND \\ LAWRENCE H. SNYDER \\ Washington University School of Medicine, St. Louis, Missouri
}

\begin{abstract}
Numerous human task-switching studies have shown that decision making that follows a task switch is slower and less accurate than that which follows a task repetition. Stoet and Snyder (2003a) tested humans and rhesus monkeys on a task-switching paradigm, but found, surprisingly, no switch costs in the monkeys. We hypothesized that the exceptional monkey behavior may have been due to the more extensive practice the monkeys received in comparison with human subjects. In the present study, we tested the hypothesis that extensive practice can abolish switch costs in humans. Four human subjects each performed 23,000 trials in a task-switching paradigm. We found that this amount of practice does not abolish switch costs.
\end{abstract}

The ability of humans to rapidly and flexibly switch from performing one task to performing another is fundamental to executive control. This flexibility comes with a price, however; switching from one task to another is more difficult than continuing to perform the same task. This phenomenon was first described by Jersild (1927), and since then, over 400 studies have replicated and extended these findings (see review by Monsell, 2003). Although important issues remain unanswered-for example, the extent to which switch costs may be driven by perceptual priming mechanisms (Logan \& Bundesen, 2003; Mayr \& Kliegl, 2003; Monsell \& Mizon, 2006) - the general phenomenon of task switching in humans is well established.

We previously tested humans and monkeys on a taskswitching paradigm (Stoet \& Snyder, 2003a). Two tasks were randomly interleaved. Each trial started with a task cue, and the subject was informed of which of the two tasks should be performed next. The task cue was followed by a stimulus, which, depending on its identity and on the task being performed on that trial, required a left or a right buttonpress. In a comparison of human and monkey performance, we found that the monkeys had little or no difficulty switching between tasks. Smaller switch costs in comparison with those seen in humans occurred only under special conditions - for example, when intertrial intervals were brief (Stoet \& Snyder, 2003a) or after ketamine administration (Stoet \& Snyder, 2006). Because the rhesus monkey is an important model of human cognition (Fuster, 2002; Terrace \& Metcalfe, 2004), this discrepancy between human and monkey task-switching performance warrants further study. It may, for example, shed light on the phylogenetic development of cognitive skills in humans.

In previous studies, we tested several hypotheses that might explain the differences between human and monkey task-switching behavior. We tested whether monkeys performed less accurately and/or more slowly than humans, and we found that monkeys perform just as well (Stoet \& Snyder, 2003a). We tested whether monkeys treat the task cues and the stimuli as all belonging to a single task. Although such a strategy is possible in principle (Logan \& Bundesen, 2004), we found no evidence for it in monkeys. On the contrary, we found that monkeys, like humans, begin preparing for a task as soon as the task cue is presented; this preparation had behaviorally distinct consequences in the two different tasks (Stoet \& Snyder, 2003b). The idea that monkeys use the task cue to prepare for a particular task was further supported in a study in which we used two perceptually distinct cues for each of the two tasks. With this design, we could differentiate between neural activity due to the cue itself and that due to the task information it conveyed. We found that individual neurons in the posterior parietal cortex encode abstract task information after the cue is presented, but before the target stimulus appears (Stoet \& Snyder, 2004).

Our behavioral studies (Stoet \& Snyder, 2003a, 2003b) demonstrated that, apart from a lack of switch costs, monkey task-switching performance is similar to that of humans. It remains to be determined whether the differences we observe in human and monkey performance are based on differences in cognitive architecture or in

G. Stoet, stoet@eye-hand.wustl.edu 
the experimental procedure. In particular, it is possible that the extensive practice that the monkeys received may have abolished switch costs. In previous studies, moderate practice (up to about 2,000 trials) in humans has been shown to reduce (but not abolish) switch costs (Kramer, Hahn, \& Gopher, 1999; Meiran, Chorev, \& Sapir, 2000; Rogers \& Monsell, 1995).

In the present study, we asked whether extensive training in humans would abolish switch costs. Four human subjects each performed at least 23,000 trials of a task-switching paradigm very similar to the one we used in the animal studies (Stoet \& Snyder, 2003a, 2003b, 2004, 2006).

In addition to analyzing switch costs, we tested whether task interference would be affected by practice. Task interference is defined here as the difficulty in responding to stimuli that are associated with different responses in different tasks (incongruent stimuli), in comparison with the difficulty in responding to stimuli that require identical responses in different tasks (congruent stimuli). A wellknown example of task interference is the Stroop effect (Stroop, 1935), which is a difficulty in naming the ink color of a word when its meaning is incongruent with its color (e.g., it is more difficult to name the ink color of the word red when it is printed in green ink than when it is printed in red ink). In the present study, half of the stimuli were congruent, and the other half of the stimuli were incongruent. Previously, we reported that trained monkeys are significantly slower in incongruent than in congruent trials, demonstrating that extensive practice does not abolish effects of task interference in monkeys (Stoet \& Snyder, 2003a). A secondary aim of this study was to determine whether interference effects in humans would be abolished by practice.

\section{METHOD}

\section{Subjects}

For the purposes of this study, we maximized the number of trials performed by each subject $(>23,000)$, not the total number of subjects. We tested two naive female subjects (Subjects 1 and 2) and the two (male) authors (Subjects 3 and 4), all of whom were between 25 and 45 years old and had normal or corrected-to-normal vision.

\section{Apparatus}

Participants performed the experiment at PCs running custom software for stimulus presentation and data collection. Standard PC keyboards were used for recording subject responses; subjects used the " $z$ " and "/" keys, which lie on the far left and far right of a U.S. keyboard, respectively.

\section{Stimuli}

All stimuli were presented on a black background. Task cues were white triangles, each side of which measured $3 \mathrm{~cm}$. An upright triangle cued the color task, and an upside-down triangle cued the orientation task (Figure 1). Target stimuli were $4 \mathrm{~cm}$ in length and $5 \mathrm{~mm}$ in width. Color stimuli varied in color ( 550 shades of red and 550 shades of green) and in orientation $\left(-10^{\circ}\right.$ to $10^{\circ}$ around $0^{\circ}$ or $90^{\circ}$ orientation in $1^{\circ}$ steps, resulting in 42 different orientations), producing a set of 46,200 possible stimuli.

\section{Procedure}

Trials were randomly interleaved, with a $500-\mathrm{msec}$ intertrial interval. Each trial started with a task cue $(250 \mathrm{msec})$, followed by a blank delay period $(400 \mathrm{msec})$ and then a target stimulus, which
A

\begin{tabular}{|c|c|c|}
\hline Event & Duration (msec) & \\
\hline Task Cue & 250 & \\
\hline Delay & 400 & \\
\hline Target Stimulus & $\sim 410$ & \\
\hline Response & & \\
\hline Intertrial Interval & & 500 \\
\hline
\end{tabular}

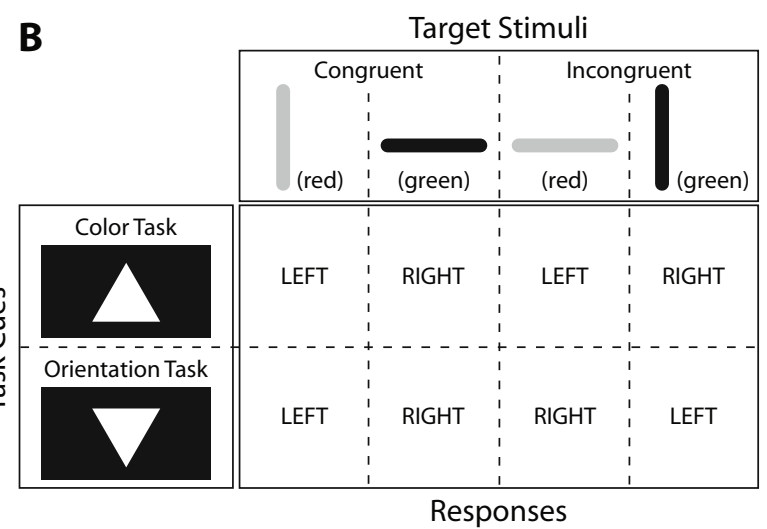

Figure 1. Task-switching paradigm. (A) The order (top to bottom) and durations in milliseconds (left to right) of events during the task-switching paradigm. Each trial started with the presentation of a task cue (upright or upside-down triangle). The cue was followed by a delay period, which was followed by the presentation of the target stimulus. The target stimulus disappeared as soon as the subject responded; thus, stimulus duration depended on reaction time. Trials were separated by 500 -msec intervals. (B) Cues, examples of target stimuli in the congruent and incongruent conditions, and the associated response location (the " $z$ " key on the far left or the "/" key on the far right of the keyboard). Target stimuli appeared in the color indicated in parentheses. Congruent target stimuli required the same response in both tasks, whereas incongruent stimuli required different responses in the two tasks. The color and line orientation of each stimulus were drawn from large and continuous distributions to prevent a simple lookup strategy.

remained on the screen until a response was made. In the color task, subjects had to press a left or right button in response to a red or green stimulus, respectively. In the orientation task, subjects had to press a left or right button in response to a horizontal or vertical stimulus, respectively.

Half of the stimuli were congruent - that is, they required the same buttonpress in both tasks. For example, a red vertical line required a left buttonpress in both the color and orientation tasks. The other half of the stimuli were incongruent, requiring opposite responses in the two tasks. For example, a red horizontal line required a left buttonpress in the color task, but a right buttonpress in the orientation task. An error message was displayed following each incorrect or slow $(>1,200 \mathrm{msec})$ response. Sessions were conducted in blocks of 168 trials. Subjects performed one to six blocks per session.

\section{Data Analysis}

We analyzed reaction time (RT) and error rate. In the RT analysis, we excluded error trials and trials immediately following an error trial. In the analysis of the error rates, we considered only those errors that followed correct trials. We used an alpha level of $5 \%$ for all statistical tests. For all data analyses, we used the statistical software package R (R Development Core Team, 2006). 
Table 1

Mean Reaction Times (RTs, in Milliseconds) and Percentages of Errors (PEs) for the Main Effects of Task Switch (Switch vs. Repeat), Congruency (Congruent vs. Incongruent), and Task (Color vs. Orientation Task)

\begin{tabular}{|c|c|c|c|c|c|c|c|c|c|c|}
\hline \multirow[b]{2}{*}{ Condition } & \multicolumn{2}{|c|}{ Subject 1} & \multicolumn{2}{|c|}{ Subject 2} & \multicolumn{2}{|c|}{ Subject 3} & \multicolumn{2}{|c|}{ Subject 4} & \multicolumn{2}{|c|}{ Difference } \\
\hline & RT & $\mathrm{PE}$ & RT & $\mathrm{PE}$ & RT & $\mathrm{PE}$ & RT & PE & RT & PE \\
\hline witch & 400 & 10 & & 8.3 & 506 & 7 & 7 & 6.3 & 2 & \\
\hline epe & 37. & 6 & 4. & & 41 & 3 & 389 & 3.1 & & \\
\hline Congruent & 374 & 4.0 & 459 & 3.2 & 448 & 2.4 & 396 & 3.9 & 14 & 6 \\
\hline Incongruent & 390 & 13.1 & 476 & 10.8 & 468 & 8.8 & 400 & 5.6 & & \\
\hline Color task & 407 & 13.6 & 457 & 5.8 & 472 & 7.9 & 403 & 6.4 & 17 & J \\
\hline Orientation task & 358 & 3.6 & 477 & 8.1 & 443 & 3.3 & 393 & 3.1 & & \\
\hline
\end{tabular}

\section{RESULTS}

We ran 25,872, 26,880, 34,944, and 23,856 trials for Subjects 1, 2, 3, and 4, respectively. Before analyzing effects of practice, we report the average RTs in the eight different conditions of the factors task (color vs. orientation), task switch (switch vs. repeat), and congruency (congruent vs. incongruent) (Table 1). We compared performance in these conditions by applying $2 \times 2 \times 2$ ANOVAs on the RT data of these eight conditions (one ANOVA for each subject). In all 4 subjects, main effects for all three experimental factors were significant $(p<.01)$. The main effect of the factor switch showed a longer average RT in the switch than in the repeat condition in all 4 subjects. All subjects were slower in the incongruent than in the congruent condition. The same pattern of data was found in the error rates: switch $>$ repeat, and incongruent $>$ congruent $\left(\chi^{2}\right.$ tests, all $\left.p s<.01\right)$. All but Subject 2 performed faster and more accurately in the orientation task.

We analyzed switch and congruency costs after extensive practice. We chose to compare subjects' performance in the last 2,500 trials (i.e., the last 15 blocks). For each subject, we performed an ANOVA on the RT data with the factors task switch and congruency, and we analyzed error data with $\chi^{2}$ tests. All 4 subjects showed highly significant switch costs in RT $(29,21,113$, and $24 \mathrm{msec}$ in Subjects $1-4$, respectively, $p<.01$; see Figure 2 ). We found significant switch costs in the error rates for all but Subject $2(p<.05)$. Similarly, we found highly significant costs of task interference (measured by comparing congruent with incongruent trials) in both RT and error rates. There were main effects of 14,22 , and $25 \mathrm{msec}$ in Subjects 1, 2, and 3, respectively (all $p$ s $<.001$ ); the results of Subject 4 were not significant. The error rates were $4.4 \%, 5.7 \%, 4.4 \%$, and $3.4 \%$ for Subjects $1,2,3$, and 4 , respectively (all $p$ s $<.001$ ). In all but Subject 1 , we found significant interactions $(p<.001)$ between the two factors, indicating smaller switch costs under congruent conditions (Table 2).

To test whether this persistence of switch and interference costs might be an artifact of the particular set of trials we chose to analyze, we repeated the same analysis using the final 1,000, 5,000, and 10,000 trials. For RT, the main effects of switch were significant for all 4 subjects, and the main effects of task interference were significant for Subjects 1, 2, and 3. For error rate, the main effects of switch and interference were significant for all subjects for the last 2,500 and 10,000 trials, and for half of the subjects for the last 1,000 and 5,000 trials.

In order to visualize the effects of practice in the different conditions, we plotted switch costs against practice time (Figure 3 ). The data were fit with a nonlinear smoothing function, Lowess, in order to visualize the time course of switch costs on a coarse time scale (thousands of
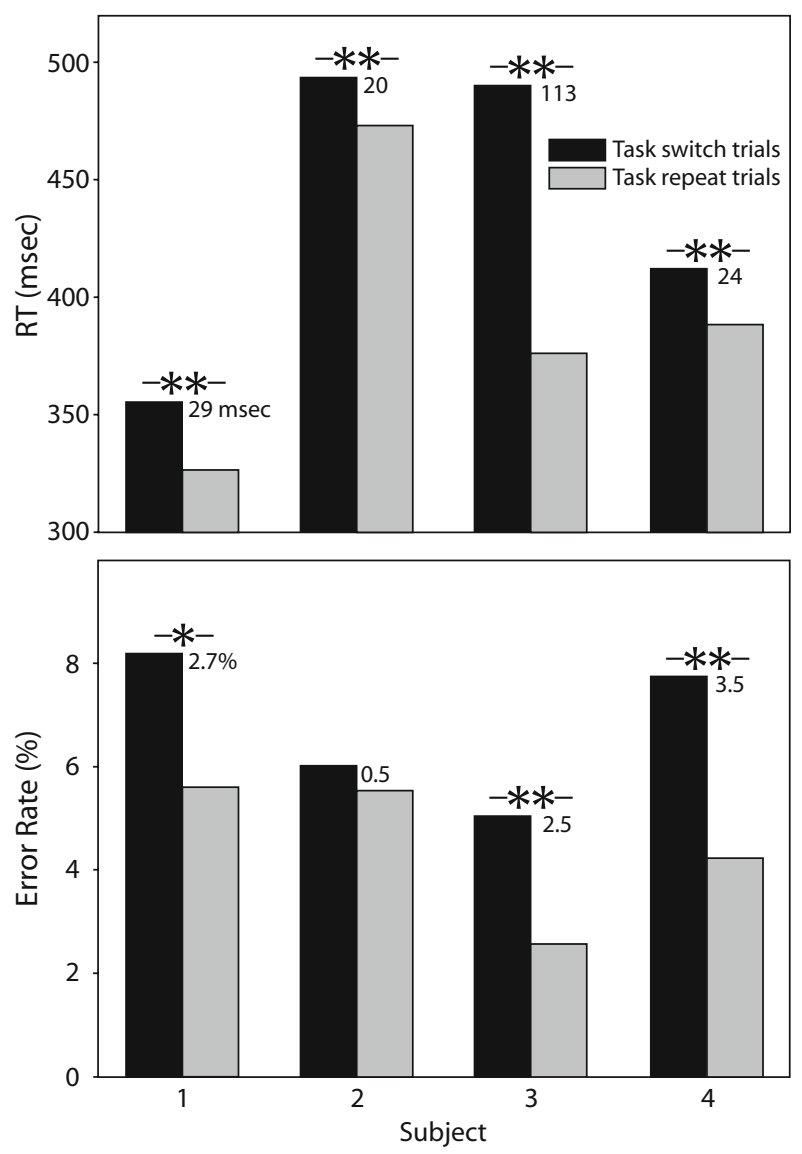

Figure 2. Switch costs after more than 20,000 trials of practice. For each subject, the mean reaction time (RT) and error rate on task switch and task repeat trials are shown for the final 2,500 trials. The black bars (switch trials) are significantly higher than the gray bars (repeat trials). Standard error for each RT bar was between 1 and $3 \mathrm{msec}$ and is therefore not displayed. Similar results were found when the final $1,000,5,000$, or 10,000 trials were analyzed. ${ }^{*} p<.05$. ${ }^{* *} p<.01$. 
Table 2

Mean Reaction Times (RTs, in Milliseconds) and Percentages of Errors (PEs) by Condition in the Final 2,500 Trials of Each Subject

\begin{tabular}{|c|c|c|c|c|c|c|c|c|}
\hline \multirow[b]{2}{*}{ Condition } & \multicolumn{2}{|c|}{ Subject 1} & \multicolumn{2}{|c|}{ Subject 2} & \multicolumn{2}{|c|}{ Subject 3} & \multicolumn{2}{|c|}{ Subject 4} \\
\hline & RT & $\mathrm{PE}$ & RT & $\mathrm{PE}$ & RT & $\mathrm{PE}$ & RT & $\mathrm{PE}$ \\
\hline Switch-congruent & 348 & 6.5 & 476 & 3.9 & 467 & 1.9 & 407 & 5.2 \\
\hline Switch-incongruent & 363 & 10.0 & 511 & 8.0 & 513 & 8.1 & 418 & 10.4 \\
\hline Repeat-congruent & 319 & 2.9 & 470 & 1.9 & 374 & 1.3 & 393 & 3.4 \\
\hline Repeat-incongruent & 334 & 8.2 & 477 & 9.2 & 379 & 3.8 & 383 & 5.1 \\
\hline
\end{tabular}

trials). The fits demonstrate that, at this scale, switch costs were roughly constant in one naive subject (Subject 1), decreased in the second naive subject (Subject 2), and showed small increases in the experienced subjects (Subjects 3 and 4). In the 1 subject who showed a decrease in switch costs, this decrease did not extend throughout the entire duration of practice. Instead, switch costs reached a plateau after about 15,000 trials, and this plateau was maintained over the final 12,000 trials.

On a finer time scale, there was a clear initial drop in switch costs in the 2 naive subjects. In these 2 subjects, switch costs in the first block of trials were large [means (and standard errors) of 135 (26) and 75 (30) msec, respectively] and decreased rapidly over approximately the next 1,200 trials.

To more closely examine the behavior of switch costs over time-and, specifically, to test the hypothesis that gradual reductions in switch costs might persist throughout training and still be occurring even after tens of thousands of practice trials - we applied a linear regression analysis to the block-by-block cost of switching on RTs. We tested the last 2,500 trials (15 blocks) and found no significant negative linear component in the data from any of the subjects. Again, to test whether this effect could be an artifact of the particular set of trials we chose to analyze, we repeated the same analysis using the final 1,000, 5,000 , and 10,000 trials. There was no significant linear component for any subject in any interval.

We performed the same analyses on the task interference data. Again, we found no significant negative linear component in any of the regressions for any of the intervals (i.e., 1,000, 2,500, 5,000, and 10,000 trials). Thus, for all subjects, we found changes in neither task-switching nor task-interference costs.
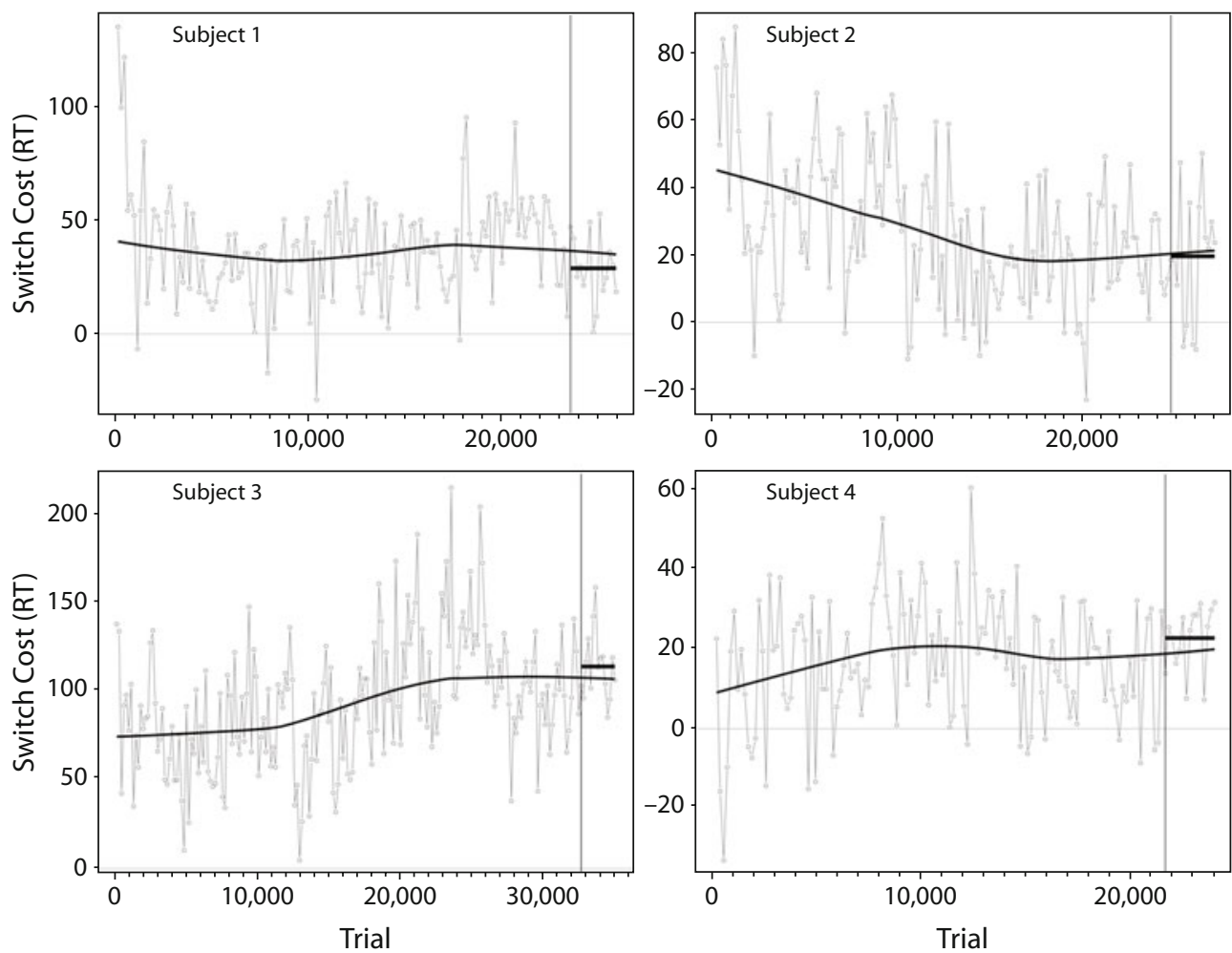

Figure 3. Switch cost as a function of practice. For each subject, the time course of switch costs (switch trial RT - repeat trial RT) is displayed. Data are calculated for 168 trial blocks (gray lines). The black curves reflect a nonlinear smoothing (the Lowess function, with a span set to two thirds). Switch costs were present up to and including the end of training, as indicated by bars showing average switch costs for each subject's final 2,500 trials. 


\section{DISCUSSION}

We measured the effects of extensive practice on taskswitching performance. We found that highly significant switch costs persisted throughout training, even after as many as 30,000 practice trials. Although naive subjects showed an initial decrease in switch costs over the first 1,000 or 2,000 trials, there was no systematic reduction in switch costs after the first 15,000 trials. Our finding that switch costs persist after tens of thousands of practice trials extends previous findings that switch costs persist after more moderate levels of practice (up to 3,000 trials; Kramer et al., 1999; Rogers \& Monsell, 1995; Stoet \& Snyder, 2003a).

It is possible that switch costs reflect a fundamental constraint of human cognitive architecture: It may be impossible to switch between two tasks without incurring a switch cost. Yet humans routinely practice many tasks or task sequences hundreds of thousands or even millions of times. For example, consider a professional basketball player who has been practicing layups since grade school. It is conceivable - but highly speculative - that part of the advantage of such extreme amounts of practice lies in minimizing or even eliminating switch costs. Although we found no significant decrease in switch costs in the final 10,000 practice trials, we cannot rule out a very small rate of decrease that might nonetheless have a substantial effect over the course of a very large number of additional trials. To further investigate this, it may be worthwhile for researchers to repeat the present study, but with 300,000 or even 3,000,000 practice trials.

As with switch costs, we found that interference costs also persist after tens of thousands of practice trials, although interference costs are smaller than switch costs. As with switch costs, task interference did not change in the final 10,000 practice trials.

\section{Interspecies Comparison}

We recently compared human and rhesus monkey performance on a switch-task paradigm and found that monkeys did not exhibit switch costs (Stoet \& Snyder, 2003a). The monkeys had received enough practice to become proficient in the task, and we speculated that the extensive practice might have abolished switch costs. The present results indicate that, even if this speculation is correct, the training by itself cannot explain the differences we observed between monkeys and humans. The design of the task was identical for the two species, and performanceincluding speed and accuracy - was very similar across species. The monkeys performed more trials than did the humans ( 100,000 vs. $\sim 25,000)$, but the monkeys learned more slowly than did the humans, and we saw no trends in the human data that would suggest that 100,000 trials in humans would lead to qualitatively different results. Also, the level of motivation is likely to have been different in the two species. Monkeys perform the task in order to obtain fluid for drinking, but humans are essentially self-motivated. We cannot rule out the possibility that the higher level of motivation might have fundamentally changed performance in the monkeys.
It seems that switch costs are not a necessary consequence of a cognitive architecture that permits switching between tasks, since monkeys are able to switch between tasks and show switch costs only under certain conditions. Instead, we hypothesize that switch costs in humans reflect a mechanism that is uniquely present in the human species to promote focus on one particular task. Such sustained focus may be of substantially greater value in humans than in monkeys, because, among primates, only human society has developed such a high level of social cooperation that an individual can focus on a specialized activity (e.g., the construction of tools) to the exclusion of all else (e.g., monitoring the surroundings for predators). Thus, switch costs may reflect a phylogenetic mechanism for decreasing distractibility.

In summary, extensive practice (tens of thousands of trials) does not abolish switch costs in humans. Trained monkeys, however, do not exhibit switch costs. Our data suggest that this represents a true species difference and not a practice effect. We argue that a general theory of task switching should offer an explanation for this speciesspecific difference. It seems unlikely that humans suffer from an undesirable cost in task switching that monkeys completely avoid. We hypothesize instead that human switch costs reflect an evolutionarily advantageous cognitive mechanism that helps humans maintain focused attention on a particular task for long periods of time.

\section{AUTHOR NOTE}

This study was supported by the NIH (NEI and Silvio Conte Center). We thank Hannes Ruge for commenting on drafts of this article. Correspondence concerning this article should be addressed to G. Stoet, Institute of Psychological Sciences, University of Leeds, Leeds LS2 9JT, England (e-mail: stoet@eye-hand.wustl.edu).

\section{REFERENCES}

Fuster, J. M. (2002). Cortex and mind: Unifying cognition. Oxford: Oxford University Press.

Jersild, A. T. (1927). Mental set and shift. Archives of Psychology, 14 (Whole No. 89).

Kramer, A. F., Hahn, S., \& Gopher, D. (1999). Task coordination and aging: Exploration of executive control processes in the task switching paradigm. Acta Psychologica, 101, 339-378.

LogAN, G. D., \& BundesEN, C. (2003). Clever homunculus: Is there an endogenous act of control in the explicit task-cuing procedure? Journal of Experimental Psychology: Human Perception \& Performance, 29, 575-599.

Logan, G. D., \& Bundesen, C. (2004). Very clever homunculus: Compound stimulus strategies for the explicit task-cuing procedure. Psychonomic Bulletin \& Review, 11, 832-840.

MAYr, U., \& KLIEGL, R. (2003). Differential effects of cue changes and task changes on task-set selection costs. Journal of Experimental Psychology: Learning, Memory, \& Cognition, 29, 362-372.

Meiran, N., Chorev, Z., \& Sapir, A. (2000). Component processes in task switching. Cognitive Psychology, 41, 211-253.

Monsell, S. (2003). Task switching. Trends in Cognitive Sciences, 7, 134-140.

Monsell, S., \& Mizon, G. A. (2006). Can the task-cuing paradigm measure an endogenous task-set reconfiguration process? Journal of Experimental Psychology: Human Perception \& Performance, 32, 493-516.

R Development Core Team (2006). R: A language and environment for statistical computing [Computer software]. Vienna: R Foundation for Statistical Computing.

Rogers, R. D., \& Monsell, S. (1995). Cost of a predictable switch 
between simple cognitive tasks. Journal of Experimental Psychology: General, 124, 207-231.

Stoet, G., \& Snyder, L. H. (2003a). Executive control and taskswitching in monkeys. Neuropsychologia, 41, 1357-1364.

Stoet, G., \& SNyder, L. H. (2003b). Task preparation in macaque monkeys (Macaca mulatta). Animal Cognition, 6, 121-130.

Stoet, G., \& SNyder, L. H. (2004). Single neurons in posterior parietal cortex of monkeys encode cognitive set. Neuron, 42, 1003-1012.

Stoet, G., \& SNyder, L. H. (2006). Effects of the NMDA antagonist ketamine on task-switching performance: Evidence for specific impairments of executive control. Neuropsychopharmacology, 31, $1675-1681$.

Stroop, J. R. (1935). Studies of interference in serial verbal reactions. Journal of Experimental Psychology, 18, 643-662.

Terrace, H. S., \& Metcalfe, J. (2004). The missing link in cognition: Origins of self-reflective consciousness. Oxford: Oxford University Press.

(Manuscript received November 24, 2005; revision accepted for publication November 20, 2006.) 